\title{
Psychometric properties of Persian Diabetes-Mellitus Specific Quality of Life (DMQoL) questionnaire in a population-based sample of Iranians
}

\author{
Mohsen Saffari ${ }^{1,2} \cdot$ Chung-Ying $\operatorname{Lin}^{3} \cdot$ Keisha O'Garo ${ }^{4} \cdot$ Harold G. Koenig ${ }^{5,6,7} \cdot$ Hormoz Sanaeinasab ${ }^{2}$. \\ Amir H. Pakpour ${ }^{8,9}$ (1)
}

Received: 2 January 2018 / Accepted: 19 May 2018

(C) Research Society for Study of Diabetes in India 2018

\begin{abstract}
Health-related quality of life (HRQoL) among patients with diabetes mellitus is often poorer than in those with other chronic medical conditions. Appropriate disease specific measures are needed to measure HRQoL in these patients. This study sought to validate a culturally adapted version of the Diabetes-Mellitus Specific Quality of Life (DMQoL) questionnaire module in Persian. Concurrent validity of the scale was assessed by the Diabetes Quality of Life (DQOL) questionnaire. Convergent and discriminative validity of the DMQoL was determined using a brief version of World Health Organization's Quality of Life Scale Brief version (WHOQOL-BREF), Hospital Anxiety and Depression Scales (HADS), and Medication Adherence Report Scale (MARS). Construct validity was examined using confirmatory factor analysis. Rasch analysis was also performed to examine the unidimensionality of the DMQoL. Known-group method was used to examine the ability of the scale to differentiate between different categories of patients. A sample of 824 patients (512 females) with diabetes mellitus was recruited from diabetic care centers located in Qazvin, Iran. The mean age of participants was 54.1 (SD 6.3) and 27\% were smokers. All items loaded on a single factor (factor loadings $\geq 0.6$ ) and internal consistency of the scale was acceptable $(\alpha=0.89)$. Significant associations were found between the scale and DQOL, indicating concurrent validity $(p<0.001)$. The DMQoL was able to differentiate subgroups of patients with hypertension, HbAlc, cholesterol, and diabetic diet. All items were appropriate with regard to difficulty level and confirmatory factor analysis verified the scale's single dimension $(\mathrm{CFI}=0.927$; RMSEA $=0.067)$. Persian DMQoL is a reliable and valid measure of HRQoL in a Persian-speaking population with type II diabetes. Further assessment is needed to confirm the psychometric properties of the scale in other cultures and languages. Future studies are needed to determine the sensitivity of the scale to change over time in response to treatment.
\end{abstract}

Keywords Diabetes mellitus $\cdot$ Health-related quality of life $\cdot$ Psychometrics $\cdot$ Validity $\cdot$ Reliability

Amir H. Pakpour

pakpour_amir@yahoo.com

Mohsen Saffari

m.saffari@bmsu.ac.ir; Saffari.CHES@gmail.com

Chung-Ying Lin

cylin36933@gmail.com

Keisha O'Garo

keisha.ogaro@duke.edu

Harold G. Koenig

Harold.Koenig@duke.edu

Hormoz Sanaeinasab

sanain20@yahoo.co.in

1 Health Research Center, Life Style Institute, Baqiyatallah University of Medical Sciences, Tehran, Iran
2 Health Education Department, School of Health, Baqiyatallah University of Medical Sciences, Tehran, Iran

3 Department of Rehabilitation Sciences, The Hong Kong Polytechnic University, Hung Hom, Hong Kong

4 Division of Psychology, Department of Psychiatry, Duke University Medical Center, Durham, NC, USA

5 Duke University Medical Center, Durham, NC, USA

6 King Abdulaziz University, Jeddah, Saudi Arabia

7 Ningxia Medical University, Yinchuan, China

8 Social Determinants of Health Research Center (SDH), Qazvin University of Medical Sciences, Qazvin, Iran

9 Department of Nursing, School of Health and Welfare, Jönköping University, Jönköping, Sweden 